\title{
Localization of Mobile Robot using Odometry, Camera Images and Extended Kalman Filter
}

\author{
G. $\operatorname{AtAli}^{a}$, Z. GARIP ${ }^{b, *}$, D. KARAYEL ${ }^{c}$ AND S.S. OZKAN ${ }^{c}$ \\ ${ }^{a}$ Sakarya University, Mechatronics Program, 54100 Sakarya, Turkey \\ ${ }^{b}$ Sakarya University, Computer Programming, 54100 Sakarya, Turkey \\ ${ }^{c}$ Sakarya University, Department of Mechatronics Engineering, 54100 Sakarya, Turkey
}

\begin{abstract}
In this study, unlike in other similar studies, the localization of mobile robots has been realized using an integrated system based on odometry, camera images and the extended Kalman filter. This integrated system was developed to determine the location of the mobile robots in the environment. It is controlled by an interface, designed specifically for this study, based on Robot Operation System. In the designed system firstly the images have been acquired from the overhead camera located in a fixed position. The received images have been processed by image processing algorithm. This way the position and rotation angle of the mobile robot have been measured. The location and the rotation angle have been also estimated using the odometric sensors and the kinematic model of mobile robot. The data obtained by these two methods have been used in extended Kalman filter to achieve a more accurate localization of the mobile robot. In the expanded Kalman filter, the image processing system performs the function of measurement model while the odometric sensors perform the function of state forecasting model. The accuracy of the system and performance have been tested with realistic experiments. The results has shown that the developed new system can be used in professional mobile robot applications.
\end{abstract}

DOI: 10.12693/APhysPolA.134.204

PACS/topics: extended Kalman filter (EKF), mobile robot, ROS, localization

\section{Introduction}

The localization of mobile robots has become one of the most frequently encountered problems in indoor and outdoor environments. There are some suggestions for solving this problem. Mobile robot positions often can not be directly measured. Therefore, the position measurements of mobile robots has to be correctly determined by combining data received from multiple sensors. Multiple sensor approaches are particularly used in different areas such as automatic cranes, autonomous vehicles, wheelchairs and autonomous mobile driving platforms.

Odometry sensors which provide data about the position and behavior of a robot are usually used in positioning of mobile robots. However, these data are not precise enough and noisy. Therefore, the employment of odometry sensors alone is not appropriate. The position and behavior of a mobile robot can be predicted via the multi-sensor approach using data obtained from such equipment as encoders, gyroscopes, GPS receivers, laser range finder, magnetic sensors, kinect sensors, etc.

Researchers have used many of these sensors to perform mapping and localization operations [1-3]. Kalman filters and variants are generally used to improve the prediction results of mobile robots indoor/outdoor motion. Fernández et al. presented a new approach for positioning and guiding of mobile robots in indoor environments. They used an odometric system in conjunction with a

*corresponding author; e-mail: zbatik@sakarya.edu.tr
Kalman filter for the positioning of a robot. The cameras were also used to receive the visual information [4].

Santana et al. applied the Kalman filter to the data obtained from their mobile robots. Subsequently, data was processed using the Hough algorithm in order to increase the precision of the positioning of a robot [5].

The multiple sensor approaches are often used to estimate the positions of moving objects. However, this approach cannot yield the desired satisfactory performance if high accuracy position data is required. In this study, a control system that can accurately determine the position of the mobile robots has been developed. User interface has been integrated into the system in order to facilitate its usage.

\section{Estimation of the position of mobile robot based on extended Kalman filter}

Kalman filter has become popular in robotics with the technological advances. With this method, the performances of robots have increased considerably. Kalman filter is usually used in such areas as monitoring, motion control, prediction and restructuring of image sequences. It is used to solve such problems in the robotic field, as object modeling, control, goal tracking, search, identification and robotic manipulation, positioning, localization, mapping, navigation, etc. [6].

Extended Kalman filter (EKF) has been used in this study because the variables in the study are independent of each other. The EKF is used because it represents a nonlinear model. In this study a ROS-based (Robot Operation System) robot called Kobuki was used. It is shown in Fig. 1. MATLAB was used to provide control of the robot and to process images from a web camera, monitoring the robot's workspace. 


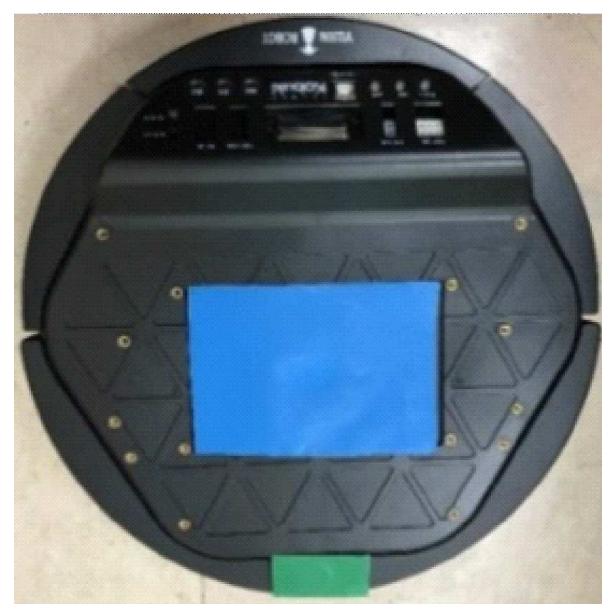

Fig. 1. Kobuki robot.

A computation model was established with the image processing algorithm, developed in the study. A blue tag was fixed to the center of the robot for locating and a green tag was fixed to the front side of the robot for determination of its direction, as seen in Fig. 1. These tags are used for the determination of the position and the angle of rotation of the mobile robot in the coordinate system of the camera.

Firstly, the positions of these blue and green tags are transformed into coordinates of the camera coordinate system $(x, y)$ using the image processing algorithm. The workspace with dimensions of $1.60 \times 2 \mathrm{~m}$ has been converted into pixel form during the processing. The positions of the blue tag $\left(x_{\mathrm{b}}, y_{\mathrm{b}}\right)$ and the green tag $\left(x_{\mathrm{g}}, y_{\mathrm{g}}\right)$ have been used to determine the angle of rotation of the robot using Eq. (1).

$$
\alpha=\tan ^{-1}\left(\frac{y_{\mathrm{b}}-y_{\mathrm{g}}}{x_{\mathrm{b}}-x_{\mathrm{g}}}\right) .
$$

\section{Calculation of position and rotation angle based on odometry}

Essentially odometry is a classic method used to calculate the position of a robot. The kinematic model of the mobile robot includes wheel information, axis $(x, y)$ and rotation angle $\alpha$, as shown in Fig. 2. The movement of the mobile robot depends on the speed of the driving wheels, which are independent of each other.

Kinematic model of differential-driven mobile robot, used in the study is determined by Eqs. (2)-(7). The description of the values in these equations are given in Table I.

$$
\begin{aligned}
& v_{\mathrm{r}}=\frac{2 v b \omega}{l}, \\
& v_{\mathrm{l}}=\frac{2 v-b \omega}{2}, \\
& s_{\mathrm{l}}=v_{\mathrm{l}} d_{\mathrm{t}},
\end{aligned}
$$

$$
\begin{aligned}
& s_{\mathrm{r}}=v_{\mathrm{r}} d_{\mathrm{t}}, \\
& \delta_{\alpha}=\omega \delta_{\mathrm{t}}, \\
& I_{\mathrm{cc}}=[x-R \sin (\alpha), y+R \cos (\alpha)] .
\end{aligned}
$$

TABLE I

Nomenclature of values used in Equations (2)-(7).

\begin{tabular}{l|l}
\hline \hline$v_{\mathrm{r}}$ & Right wheel velocity \\
$v_{1}$ & Left wheel velocity \\
$b$ & Wheelbase \\
$x, y$ & Location \\
$w$ & Angular velocity \\
$s_{\mathrm{r}}$ & Right wheel travel distance \\
$s_{1}$ & Left wheel travel distance \\
$\delta_{\alpha}$ & Rotation angle change \\
$\delta_{\mathrm{t}}$ & Time interval \\
$I_{\mathrm{cc}}$ & Instantaneous center of curvature
\end{tabular}

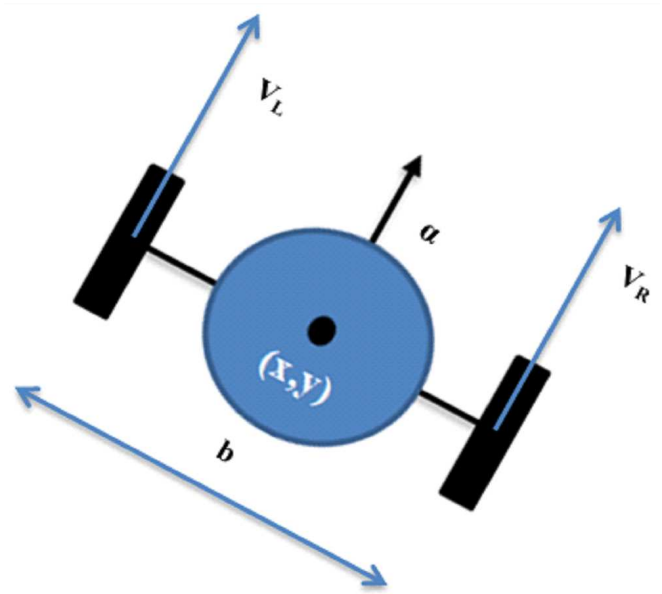

Fig. 2. Kinematic model of mobile robot.

\section{Combining data using extended Kalman filter}

The Kalman filter is one of the most commonly used methods to combine data from various sensors. In the other words, EKF is the method which is often used for estimation in nonlinear studies [7]. Data obtained from the odometry are mostly correct for short-term use. In the case of long-term use, this data is supported by various methods, such as Kalman filter method. Another method used in localization of mobile robots is image processing. As shown in Fig. 3, in this study, EKF has been applied by combining the data obtained by both methods.

Vector $x_{k}$ was obtained from odometry, vector $y_{k}$ was obtained from image processing and vector of $u_{k}$ state model is given in Eq. (8). 


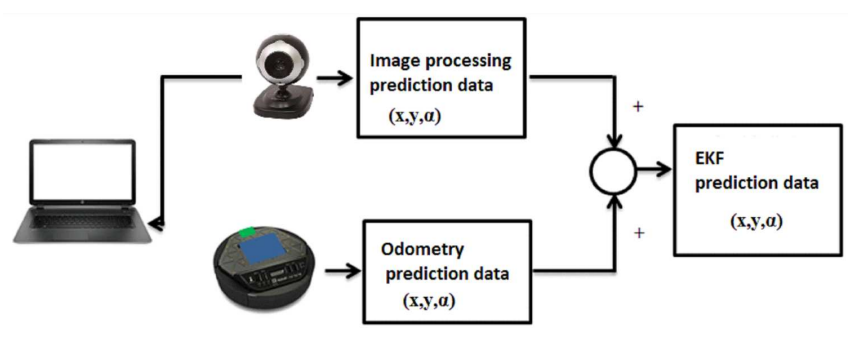

Fig. 3. Combination of designed model with EKF.

$$
x_{k}=\left[\begin{array}{l}
x_{k} \\
y_{k} \\
\alpha_{k}
\end{array}\right], \quad y_{k}=\left[\begin{array}{l}
x_{k} \\
y_{k} \\
\alpha_{k}
\end{array}\right], \quad u_{k}=\left[\begin{array}{l}
v_{\mathrm{r}} \times \delta_{\mathrm{t}} \\
v_{\mathrm{l}} \times \delta_{\mathrm{t}}
\end{array}\right] .
$$

The state-space model of wheel velocities is given in Eq. (10).

$$
\begin{aligned}
& {\left[\begin{array}{l}
x_{\mathrm{t}+\delta_{\mathrm{t}}} \\
y_{k+\delta_{\mathrm{t}}} \\
\alpha_{k+\delta_{\mathrm{t}}}
\end{array}\right]=\left[\begin{array}{ccc}
\cos \left(\omega \delta_{\mathrm{t}}\right) & -\sin \left(\omega \delta_{\mathrm{t}}\right) & 0 \\
\sin \left(\omega \delta_{\mathrm{t}}\right) & \cos \left(\omega \delta_{\mathrm{t}}\right) & 0 \\
0 & 0 & 1
\end{array}\right]\left[\begin{array}{c}
x-I c_{x} \\
y-I c_{y} \\
0
\end{array}\right]} \\
& +\left[\begin{array}{l}
I c_{x} \\
I c_{y} \\
\omega \delta_{\mathrm{t}}
\end{array}\right] .
\end{aligned}
$$

The system vector is obtained using the Eqs. (8) and (9).

$$
\begin{aligned}
& {\left[\begin{array}{l}
x_{\mathrm{t}+\delta_{\mathrm{t}}} \\
y_{\mathrm{t}+\delta_{\mathrm{t}}} \\
\alpha_{\mathrm{t}+\delta_{\mathrm{t}}}
\end{array}\right]=f\left(x_{\mathrm{t}}, y_{\mathrm{t}}, \alpha_{\mathrm{t}}, s_{\mathrm{r}}, s_{\mathrm{l}}\right)=} \\
& {\left[\begin{array}{l}
x_{\mathrm{t}} \\
y_{\mathrm{t}} \\
\alpha_{\mathrm{t}}
\end{array}\right]+\left[\begin{array}{c}
\frac{b\left(s_{\mathrm{r}}+s_{1}\right)\left[\sin \left(\alpha+\delta_{\alpha}\right)-\sin (\alpha)\right]}{2\left(s_{\mathrm{r}}-s_{1}\right)} \\
\frac{b\left(s_{\mathrm{r}}+s_{1}\right)\left[-\cos \left(\alpha+\delta_{\alpha}\right)+\cos (\alpha)\right]}{2\left(s_{\mathrm{r}}-s_{1}\right)} \\
\delta_{\alpha}
\end{array}\right] .}
\end{aligned}
$$

The pseudo code for the extended Kalman filter is given below.

$$
\begin{aligned}
& x_{0}=\{0\}, \quad P_{0}^{+}=\{0\}, \quad Q=\{\}, \quad R=\{\} \\
& \text { while } \\
& \{\text { Previous estimate }\} \text { - linearization } \\
& x_{k}^{-}=f\left(x_{k-1}, s_{\mathrm{r}}, s_{1}\right) \\
& P_{k}^{-}=A P_{k-1} A^{T}+Q \\
& \{\text { Measurement update - next estimate }\} \text { - linearization } \\
& \text { if (value of measurement comes from the image } \\
& \text { processing) } \\
& K_{k}=P_{k}^{-} C^{T}\left(C P_{k}^{-} C^{T}+R\right)^{-1} \\
& \hat{x}_{k}=x_{k}^{-}+K_{k}\left(y_{k}-C x_{k}^{-}\right) \\
& P_{k}=\left(I-K_{k} C\right) P_{k}^{-} \\
& \text {else } \\
& P_{k}^{+}=P_{k}^{-}, \quad x_{k}^{+}=x_{k}^{-} \\
& \text {end if } \\
& \text { Output calculation } \\
& y_{k}=C_{k} x_{k}+v_{k}
\end{aligned}
$$

In the experimental study, $Q$ matrix is used as process covariance, while $R$ matrix is used as measurement covariance. The noise read from the encoders of the mobile robot and the measurement from the image processing have been determined in $Q$ and $R$ matrices.
The noise read in the $Q$ matrix, the process noise, is defined as $n_{\mathrm{p}}, 2 \delta_{\mathrm{t}}(\mathrm{m} / \mathrm{s})$ and measurement noise in $R$ matrix is defined as $n_{\mathrm{m}}, 0.1 \mathrm{~m}$.

$$
Q=\left[\begin{array}{ccc}
n_{\mathrm{p} x}^{2} & 0 & 0 \\
0 & n_{\mathrm{p} y}^{2} & 0 \\
0 & 0 & n_{\mathrm{p} \theta}^{2}
\end{array}\right], \quad R=\left[\begin{array}{ccc}
n_{\mathrm{m} x}^{2} & 0 & 0 \\
0 & n_{\mathrm{m} y}^{2} & 0 \\
0 & 0 & 0
\end{array}\right]
$$

\section{Designed interface based on MATLAB and ROS}

In this study, a graphical interface has been designed to determine the position of the robot and the angle of rotation using the MATLAB-GUI. With the help of the designed interface, ROS, camera and odometry data could be combined and used effectively. In addition to being an operating system, ROS can be thought of as a layer between robot hardware and software. Different robot platforms can be easily controlled by means of the libraries and services defined in this layer. In addition to these features, it also offers code development environment. Robot behavior algorithms are published to the simulation program of the robot in ROS via the program parts called nodes. MATLAB has many graphical functions as well as mathematical capabilities and can actively use all operations of the ROS.

The developed interface consists of six parts: connect/disconnect to robot, manual control, extended Kalman filter, imaging, image processing, graphic interface. The example of the position estimation of the mobile robot using EKF imaging, EKF odometry and the combination of them, is given in Fig. 4.

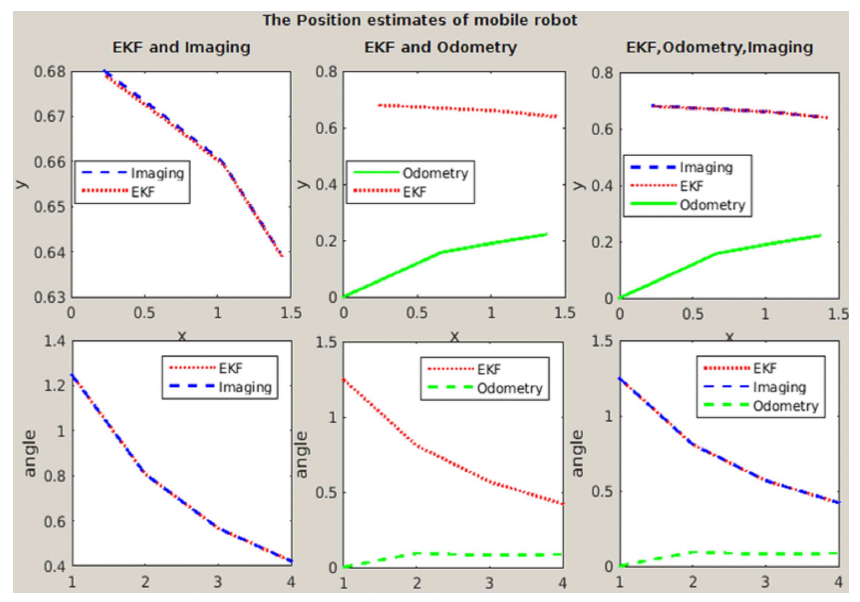

Fig. 4. The position estimates of mobile robot.

The measurements provided by image processing have been used in the measurement model and odometry data have been used in the state model during mobile robot motion. The data obtained from the measurement model and the state model have been combined with the EKF to estimate the position of the mobile robot.

Results are presented simultaneously in the GUI. The dotted blue line corresponds to image processing data, 
green line corresponds to odometry data, and dotted red line corresponds to data estimated by EKF. While odometry is in use, it causes the accumulation of errors over time due to such reasons as wheel slip, mechanical tolerance and surface roughness. It has been observed that the EKF system gives better results than the image processing system. Therefore, the proposed model is provided with effective internal locating with low error. The comparison of these cases is given in Table II.

Resulting values of positioning by imaging, odometry and EKF combination.

TABLE II

\begin{tabular}{|c|c|c|c|c|c|c|c|c|}
\hline \multicolumn{3}{|c|}{ Imaging $[\mathrm{m}]$} & \multicolumn{3}{|c|}{ Odometry $[\mathrm{m} / \mathrm{s}]$} & \multicolumn{3}{|c|}{$\mathrm{EKF}[\mathrm{m}]$} \\
\hline$x$ & $y$ & $\alpha$ & $x$ & $y$ & $\alpha$ & $x$ & $y$ & $\alpha$ \\
\hline 0.2300 & 0.6800 & 1.2463 & 0 & 0 & 0 & 0.2440 & 0.6788 & 1.2463 \\
\hline 0.6400 & 0.6700 & 0.8085 & 0.6668 & 0.1582 & 0.0935 & 0.6540 & 0.6690 & 0.8085 \\
\hline 1.0300 & 0.6600 & 0.5682 & 1.0267 & 0.1914 & 0.0792 & 1.0440 & 0.6591 & 0.5682 \\
\hline 1.4200 & 0.6400 & 0.4214 & 1.3727 & 0.2211 & 0.0852 & 1.4344 & 0.6391 & 0.4214 \\
\hline
\end{tabular}

\section{Conclusions}

In this study the data obtained using the camera and the odometry sensors were combined with the EKF to ensure the correct localization of the robot in the known environment. As a result of the study, odometry data are insufficient for localization of mobile robots. For this reason, odometry data should be supplemented with EKF and its variants. The positioning in the study has been achieved by combining the data obtained from the camera and the odometry sensors. In addition, EKF has been applied to these data and the estimation data has become more accurate. As a result, the localization was made more accurately with combination of odometry, imaging and EKF.

\section{References}

[1] J. Lategahn, F. Kuenemund, C. Roehrig, Int. J. Comput. 9, 22 (2010).
[2] S. Karakaya, H. Ocak, G. Küçükyıldız, O. Kılınç, Proceedings Int. Conf. Image Proces., Comp. Vision, and Pattern Recognition, IPCV 2010, Las Vegas, USA 2015, p. 224.

[3] D. Pizarro, M. Mazo, E. Santiso, M. Marron, D. Jimenez, S. Cobreces, C. Losada, Sensors 10, 3655 (2010).

[4] I. Fernández, M. Mazo, J.L. Lázaro, D. Pizarro, E. Santiso, P. Martín, C. Losada, Auton. Robots 23 , 305 (2007).

[5] A.M. Santana, A.A.S. Sousa, R.S. Britto, P.J. Alsina, A.A.D. Medeiros, Proceedings of the Fifth Int. Conf. on Informatics in Control, Automation and Robotics, Robotics and Automation 2, Funchal, Portugal 2008.

[6] P. Goel, S.I. Roumeliotis, G.S. Sukhatme, Proceedings IEEE/RSJ Int. Conf. Intelligent Robots and Systems, Kyongju, South Korea 1999.

[7] M. Nørgaard, N. Kjølstad Poulsen, O. Ravn, IFAC Proceedings Volumes 36, 1447 (2003). 